\title{
Recognition of Printed Text Based on Hidden Markov Model
}

\section{Ghayda A.A. Al-Talib}

Armanesa Nuaman Hasson

ghaydabdulaziz@uomosul.edu.iq

University of Mosul

College of Computer Sciences and Mathematics

Received on: 26/5/2010

ABSTRACT

Automatic recognition of printed text is of high importance in modern IT applications. Recognition of text for lateen scripted language is readily in use for a long time. For cursive script languages (such as Arabic language) recognition of text is not available as a robust one with a reliable performance. More improvements still exist to reduce average of incorrect words, rather then no constraints on the limit of words of a specific language.

Numerous approaches were tried in recognition of text but recognition of Arabic text based on Hidden Markov model seems to be the most promising one because of its ability to discriminate cursive scripts.

This paper provides an off-line system to recognize printed Arabic text by using hidden Markov model with the aid of the algorithm that segment the text lines into connected parts then into characters.

By looking on the results given by the designed recognition system it is found that a recognition rate $(94.9 \%)$ can be achieved. Such rate is in the same order of rates of recognition researches viewed in previous studies. This rate can still be improved. The language used in building the system is Matlab V7.6 (R2008a).

Keywords: Character Recognition, HMM

$$
\begin{aligned}
& \text { التعرف على النص العربي المطبوع باستخدام نموذج ماركوف الخفي } \\
& \text { ارمانيسة نعمان حسون } \\
& \text { قسم علوم الحاسوب } \\
& \text { جامعة تكربيت } \\
& \text { تاريخ قبول البحث: 2010/10/25 } \\
& \text { غيداء عبد العزيز الطالب }
\end{aligned}
$$

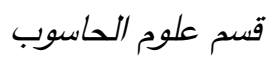

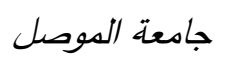

$$
\begin{aligned}
& \text { تاريخ استلام البحث: 2010/5/26 } \\
& \text { نموذج ماركوف الخفي هو الأكثر وعداً وذلك بسبب قدرته على تمييز الكتابة المتصلة. }
\end{aligned}
$$


نُقِدِم في هذا البحث نظام يعمل بأسلوب off-line للتعرف على النص العربي المطبوع باستخدام نموذج ماركوف الخفي مع الاستعانة بخوارزمية تقطيع السطر النصي إلى مقاطع ثم حروف.

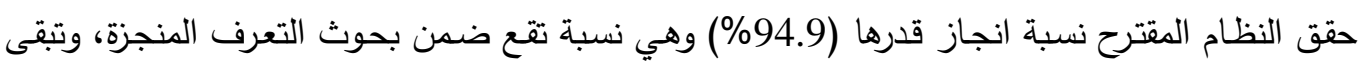
هذه النسبة قابلة للتحسين. استخدمت Matlab V7.6 (R2008a) كلغة برمجية في بناء النظام المقترح. الكلمات المفتاحية: تمييز الانماط، نموذج ماركوف الخفي

1- المقدمة

يُعد الذكاء الاصطناعي من أهم المجالات العلمية التطبيقية في علوم الحاسبات فقد تعددت وتتوعت

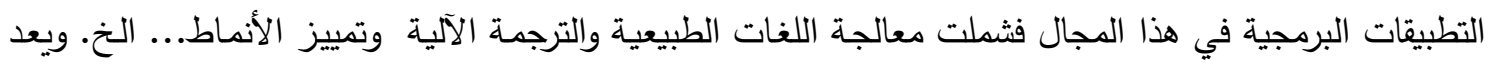

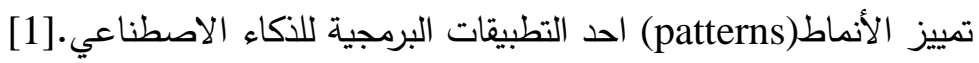
كما أن تمييز الأنماط هو دراسة كيف يُكن للمكائن أن تُلاحظ البيئة، فتتعلم إظهار أنماط ترغب بتهبة بتمييزها

وتتخذ قرارها المعقول حول أصناف تلك الأنماط. [2: وقد عرف Watanbe النمط كنظير للفوضى، بأنه كيان مُعرف بشكل غير واضـح مُككن أن يُعطى اسماً معيناً. وبالرغم من التحسينات التدريجية في تطبيقات علم تمييز الأنماط في العقد الأخير من القرن العشرين وأوائل هذا

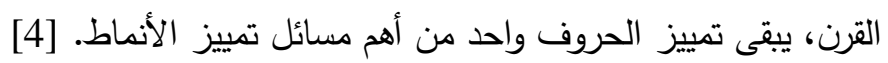

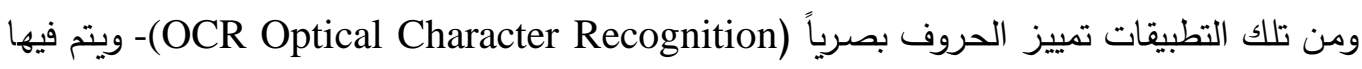
قراءة العنوان البريدي على المظروف، أرشفة واسترجاع النص، ترقيم المكتبات ... الخ. يمر OCR بعدة مراحل و

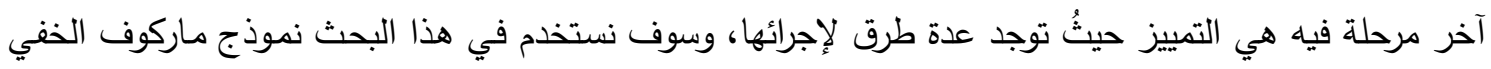
في تمييز النص العربي المطبوع. فنموذج ماركوف الخفي Hidden Markov Model-HMM) من النماذج المستخدمة في معالجة الكلام واللغات. [5] ويُعرف HMM كعملية تصـادفيه مزدوجة فيها حسالات

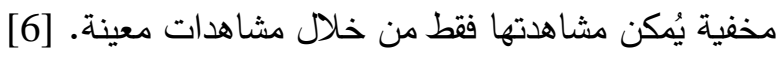
2- النموذج العام لنظام تمييز الحروف

يتكون نظام التمييز بصورة عامة من أربعة مراحل أساسية يوضحها المخطط في الثكل- 1، حيث تبدأ

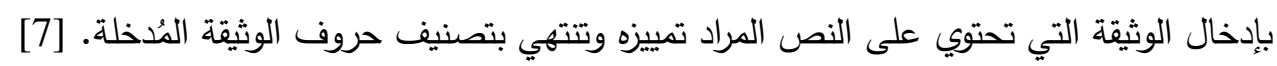




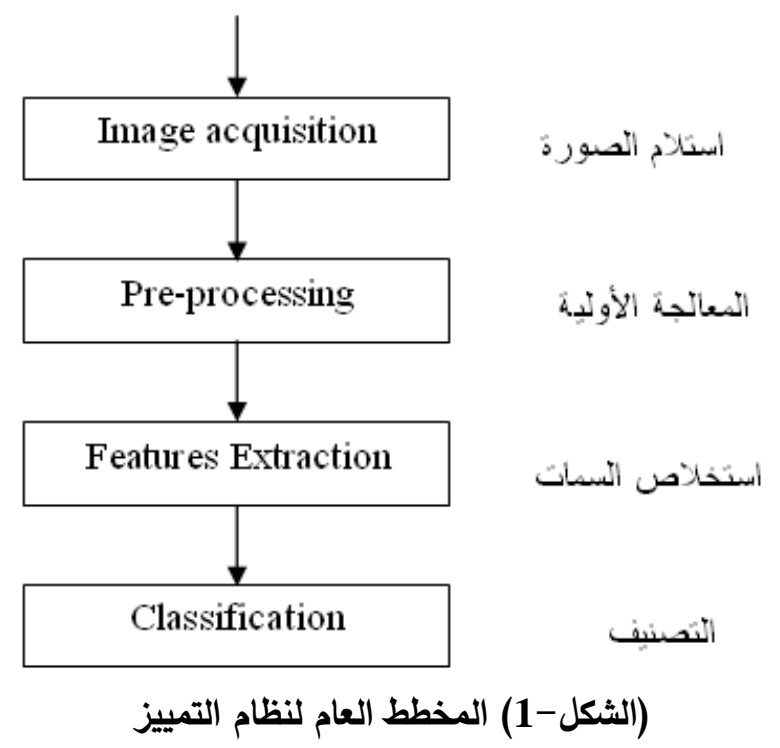

وقد لا يحتوي نظام التمييز على جميع هذه المراحل إذ يتم اختزال بعض المراحل بدون أن يؤثر ذلك على لـى

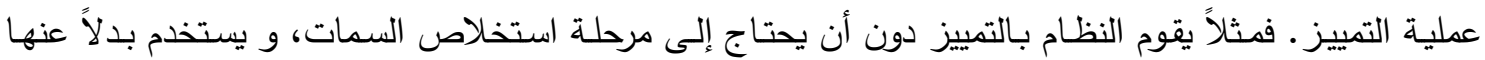
مطابقة القوالب (Templates Matching).

\section{3arkov Chains سلاسل ماركوف}

النماذج الرياضية ممكن أن تكون محددة (Deterministic) أو تصادفيه (Stochastic). ومع ذلك فأن في عدة حالات اجتماعية وحياتية هُناك ظواهر تصادفيه (وهي ظواهر ذات سلوك غير قطعي لا يمكن السيطرة عليها بشكل تام أو التتبؤ بسلوكها المستقبلي بشكل مؤكد ويُطلق عليها مصطيه وهلح العمليات التصادفية. [8] فيصبح النموذج التصادفي هو الأكثر ملائمة لتمثيلها. المنظومة الموضحة في الثكل -2 يُمكن أن توصف خلال أي فترة زمنية، كأن تكون موصوفة في واحدة من مجموعة الحالات المتقطعة)( (Discrete states) (S

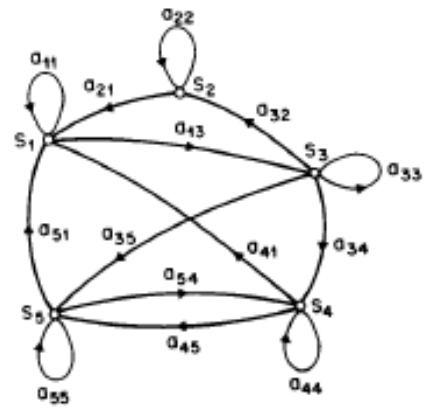

(الثكل-2) سلسلة ماركوف دـ(5) حالات مع انتقالاتها

وخلال تلك الأزمنة المتقطعة، تخضع المنظومة إلى تغيرات في الحالة (من الممكن الرجوع إلى الحالة نفسها) وفقاً لمجموعة من الاحتمالات المرتبطة بالحالة. ويُرمز إلى الزمن المرتبط بتغير الحالة بـ(....

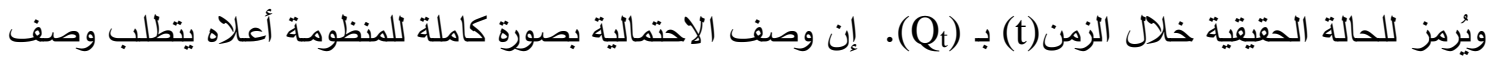
الحالة الحالية عند الزمن(t)، فضلاً عن كل الحالات السابقة لها. [6] فيُنظر إلى سلسلة ماركوف كنوع من مخطط 
الاحتمالات (Probabilistic Graphical Model) أو طريق لتمثيل الفرضيات الاحتمالية. وسلسلة ماركوف

محددة بالمكونات التالية: [5]

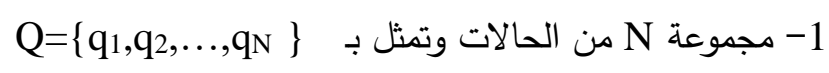

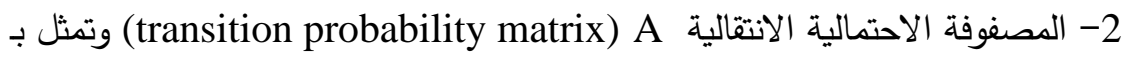

$$
\begin{aligned}
& \mathrm{A}=\left(\begin{array}{ccc}
a_{11} & \ldots & a_{1 N} \\
\cdot & \cdot & \cdot \\
\cdot & \cdot & \cdot \\
\cdot & \cdot & \cdot \\
a_{N 1} & \ldots & a_{N N}
\end{array}\right) \\
& \text { حيث أن كل aij تُمثل احتمالية الانتقال من الحالة i الى الحالة j بحيث تحقق الثرط التالي: } \\
& \forall i \quad \sum_{j=1}^{N} \mathrm{a}_{\mathrm{ij}}=1
\end{aligned}
$$

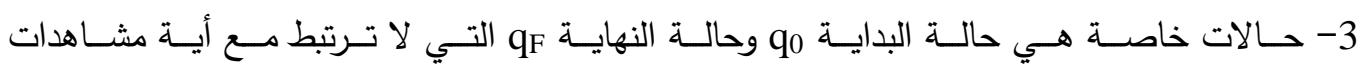

.(Observations)

4- التوزيع الاحتمالي الابتدائي على الحالات (Initial probability distribution)

$$
\pi=\pi_{1}, \pi_{2}, \ldots, \pi_{N}
$$

$$
\text { وكنلك }
$$

وتكون الاحتماليـة (probability) التي تبـدأ بها سلسـلة مـاركوف عند الحالـة i في بعض الحسالات

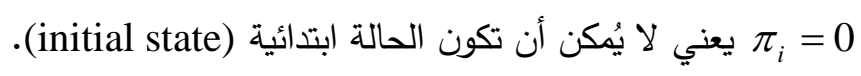

وتعرّف فرضية ماركوف بالعلاقة التالية:

$\mathrm{P}\left(\mathrm{q}_{\mathrm{i}} \backslash \mathrm{q}_{1} \ldots \mathrm{q}_{\mathrm{i}-1}\right)=\mathrm{P}\left(\mathrm{q}_{\mathrm{i}} \backslash \mathrm{q}_{\mathrm{i}-1}\right)$

$$
\text { حيثُ أن }
$$

q1...qii-1 تمثل احتمالية حدوث الحالة qu عند توفر الحالات P(qilq1...qqi-1)

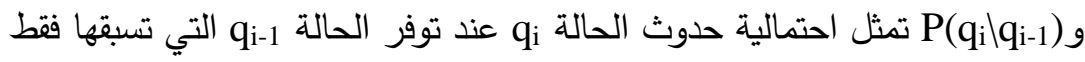

Hidden Markov Model 4- نموذج ماركوف الخفي

نموذج ماركوف الخفي (HMM) عبارة عن نظام محطات الآلة المحدودة (finite state machine) القادر على توليد مشاهدات باحتمالية انتقال الحالة عند الزمن t التي تعتمد فقط على الحالة السابقة لها عند الزمن

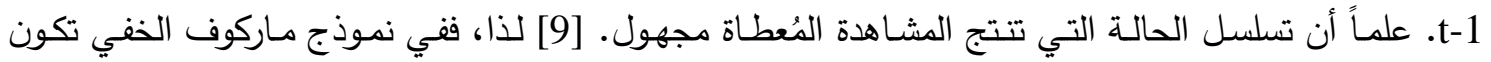
الحالة ليست مرئية، لذلك سُميَّ بنموذج ماركوف الخفي والانتقالات بين الحالات تحكمها مجموعة من الاحتمالات

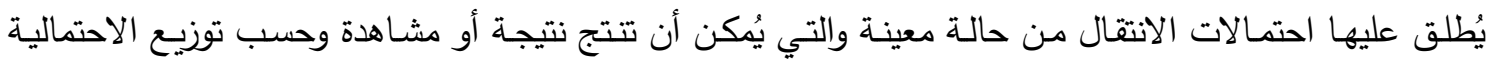
الهـرتبط بتلك الحالـة. [10] والاختلاف بـين نمـوذج مـاركوف الخفي ونمـوذج مـاركوف هـو وجـود الاحتمـالات الإضافية. ويُمثل هذا الجزء الخفي للنموذج ويرتبط بالمشاهدة الناتجة من كل حالة [11]. فنموذج ماركوف الخفي هو نموذج تصـادفي قادر على التصنيف الإحصائي. ولذلك فقد طُبِقَ في تمييز الصسوت وتمييز الكتابة اليدويـة

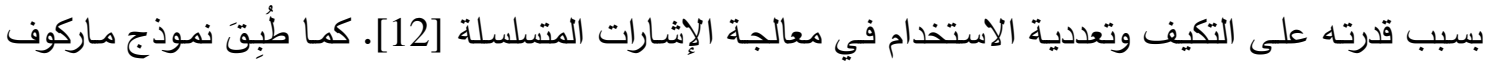
الخفي في مجتمع المعلوماتية الحياتية (Bioinformatics) لإيجاد سلاسل DNA. [13] وكذلك طُبِقَّ في تصميم 
أنظمة كثف التطفل على الثبكات (حيثُ تُعنى أنظمة كثف التطفل بحماية الثبكات من الهجمات و/أو سرقة

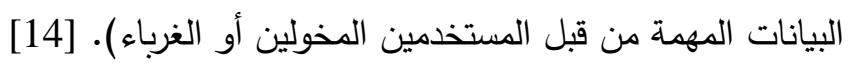

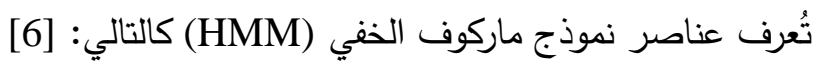
N عدد الحالات في النموذج، فبالرغم من أن الحالات مخفية إلا أن للعديد من التطبيقات الطبيعية هناك في

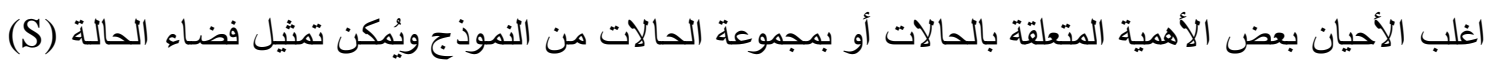

$$
\mathrm{S}=\left\{\mathrm{S}_{1}, \mathrm{~S}_{2}, \ldots, \mathrm{S}_{\mathrm{N}}\right\}
$$$$
\text { كما يلي: }
$$

$$
\text { حيثُ يُرمز للحالة عند الزمن (t) بـ (qt). }
$$

• $\mathrm{V}=\left\{\mathrm{v}_{1}, \mathrm{v}_{2}, \ldots, \mathrm{v}_{\mathrm{M}}\right\}$

$$
A=\left\{a_{i j}\right\}
$$$$
\text { • (A) التوزيع الاحتمالي للحالة الانتقالية (A): }
$$

$\mathrm{a}_{\mathrm{ij}}=\mathrm{p}\left[\mathrm{q}_{\mathrm{t}+1}=\mathrm{S}_{\mathrm{j}} \backslash \mathrm{q}_{\mathrm{t}}=\mathrm{S}_{\mathrm{i}}\right] \quad, 1 \leq i, j \leq N$

حينُ

$$
\text { • التوزيع الاحتمالي لرمز المشاهدة عند الحالة j }
$$

$$
\mathrm{B}=\left\{\mathrm{b}_{\mathrm{j}}(\mathrm{k})\right\}
$$

$$
\mathrm{B}_{\mathrm{j}}(\mathrm{k})=\mathrm{p}\left[\mathrm{v}_{\mathrm{k}} \text { at } \mathrm{t} \mid \mathrm{q}_{\mathrm{t}}=\mathrm{S}_{\mathrm{j}}\right] \quad, \quad \begin{aligned}
& 1 \leq j \leq N \\
& 1 \leq k \leq M
\end{aligned}
$$

$$
\pi=\left\{\pi_{i}\right\}
$$

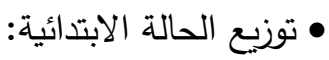

$$
\pi_{i}=p\left[q_{1}=S_{i}\right] \quad, \quad 1 \leq i \leq N
$$

وبإعطاء القيم المناسبة لكل من (N,M,A,B, $)$ يكون بالإمكان استخدام نموذج ماركوف الخفي كمولد

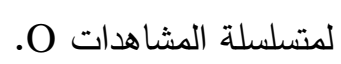

$$
\mathrm{O}=\mathrm{O}_{1} \mathrm{O}_{2} \ldots \mathrm{O}_{\mathrm{T}}
$$

$$
\text { حيثُكن أن يُمثل نموذج ماركوف الخفي بالمعلمة }
$$

$\pi$ و A تُمثل مصفوفة احتمالية انتقال الحالة. و Bُ تُثل احتمالية مشاهدة الرمز عند الحالة A

$$
\text { 5- النموذج المقترح لنظام تمييز النص العربي }
$$

يتكون نظام التمييز المقترح من مراحل أساسية تبدأ بإدخال النص المراد تمييزه وتتنهي بمرحلة التمييز

باستخدام نموذج ماركوف الخفي. والمخطط في الثكل -3 يوضح مراحل تتفيذ النظام المقترح للتمييز . 


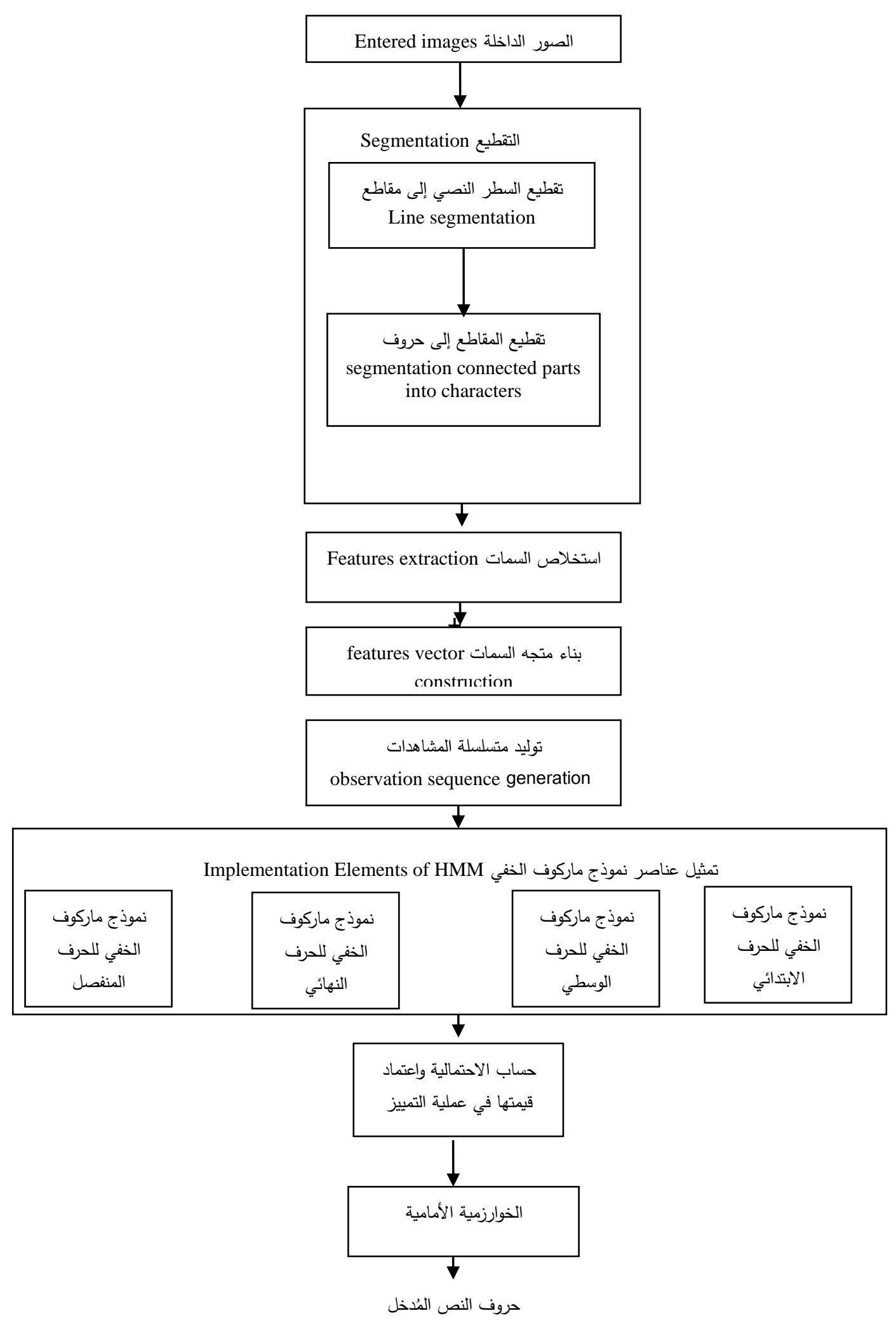

(الثكل-3) مخطط نظام التمييز المقترح 
تم في هذا البحث اقتراح خوارزميـة لتمييز النص العربي المطبوع باستخدام نـوذج مـاركوف الخفي Midden Markov Model نُفِذِت بالتعاقب لأجراء عملية التمييز (Recognition). 1-6 مرحلة التدريب يبدأ عمل النظام بمرحلة تدريب نماذج ماركوف المصممة والتي تضمنت الخطوات التالية: 1-1-6 إخال الصور تم في هذه الخطوة طباعة 28 سطراً نصياً كل سطر تمت طباعته على حدا باستخدام خط من نوع (Simplified Arabic fixed) سطر نصي حرف معين بجميع أشكاله. يبين الثكل-4 سطر واحد من 28 سطر نصي تم إدخالها.

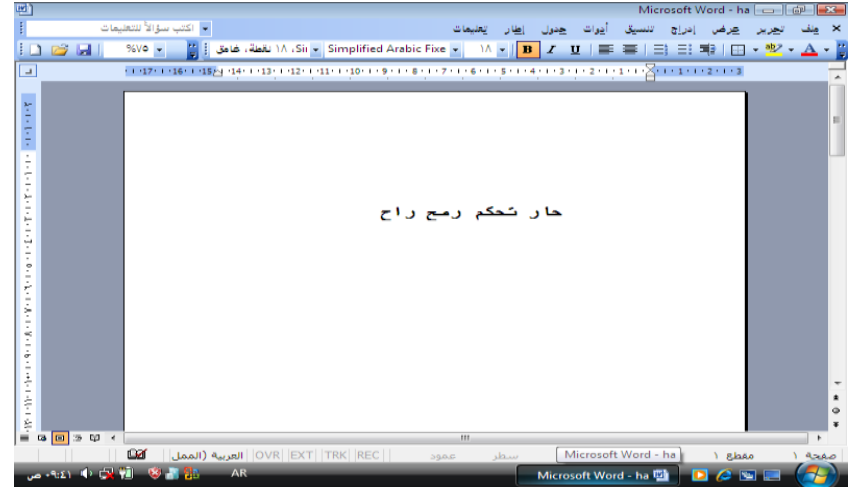

(الثكل -4) حرف الحاء بأشكاله الأربعة

وبعد ذلك يُخزن كل سطر نصي على شكل صورة ثنائية في ملف نوعه BMP. تُخزن البيانات في الصورة الثنائية بصيغة (1,0) إذ تُثل النقطة السوداء التي تكون جزءاً من النمط بالقيمة 0 والنقطة البيضـاء بالقيمة 1 والتي لا لأل تكون جزءاً من أي نمط. بعدها تُقرأ الصورة وتُخزن في مصفوفة ثنائية، ليتم بعدها إجراء العمليات اللاحقة عليها لغرض الحصول على النص المقابل للصورة. لم نُجر عملية تقليل الضوضاء بسبب عدم إدخال الصورة عن طريق أجهزة المسح البصري مثل الماسح

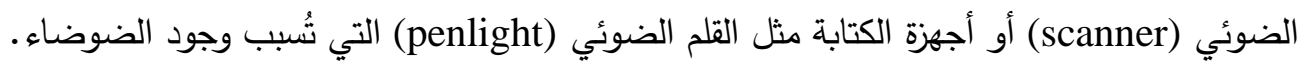

2-1-6 مرحلة التقطيع

وتعد مرحلة التقطيع مرحلة مهمة ضمن مراحل نظام تمييز النص العربي بسبب طبيعة الكتابة العربية

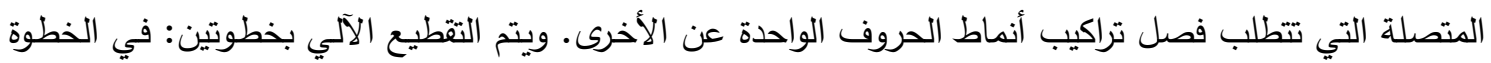
الأولى يتم تقطيع السطر النصسي إلى كلمات و/أو مقاطع وذلك باستخدام المدرج التكراري العمودي، بعدها يتم تقطيع كل كلمة و/أو مقطع إلى الحروف المكونة لها، وفيما يلي شرح تقطيع المقاطع إلى حروف: إن عملية استقطاع الحرف تتم بعد تحديد بدايته ونهايته فضلاً عن إيجاد: 1- خط الأساس Base line: يكون عند الخط line الذي يملك اكبر عدد من النقاط الضوئية السوداء. 2- الخط العلوي Top line لكل عمود في المقطع. 3- الخط السفلي Bottom line لكل عمود في المقطع. 
4- حد العتبة Threshold: يُقابل اكبر قيمة مكررة في المدرج التكراري لكل عمود الذي تم إيجاده في خطوة التقطيع السابقة.

$$
\text { 5- عدد الانتقالات العمودية من (0-1-1) أو (1-0). }
$$

عمود البداية للحرف يكون المدرج التكراري له اكبر من حد العتبة، بينما عمود النهاية يجب أن يُحقق شروط هي: أ- الخط العلوي لهذا العمود يكون اقل أو يساوي خط الأساس. ب - الخط السفلي لهذا العمود يكون اكبر من أو يساوي خط الأساس. ج- الفرق بين الخط السفلي والخط العلوي يكون اقل أو يساوي حد العتبة.

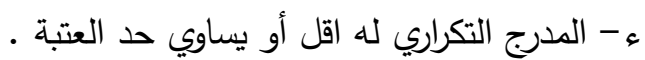
هـ - عدد الانتقالات العمودية تساوي اثثين.

و - الخط العلوي لهذا العمود يكون اكبر من الخط العلوي لعمود البداية.

3-1-6 استخلاص السمات

تم في المرحلة السابقة الوصول إلى كل حرف ومعرفة بدايته ونهايته والمساحة التي يشغلها الحرف، وفي

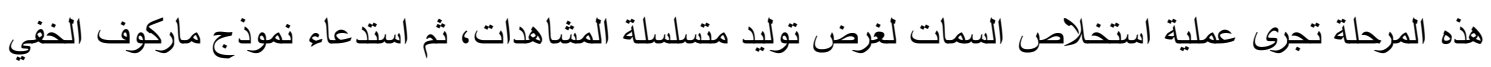
المُصمم حسب موقع الحرف، لكي يتم بعدها حساب الاحتمالية لمتسلسلة مشاهدات الحرف وإخراج الحرف المميز . وتُكرر هذه الخطوات على بقية الحروف بالتتابع.

4-1-6 بناء متجه السمات

يتكون متجهـ السمات من ثمانِ متغيرات كل متغير يمثل سمة من السمات التي وجدناها سابقاً ويكون

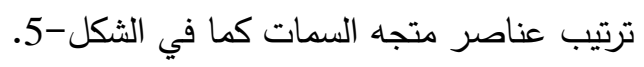

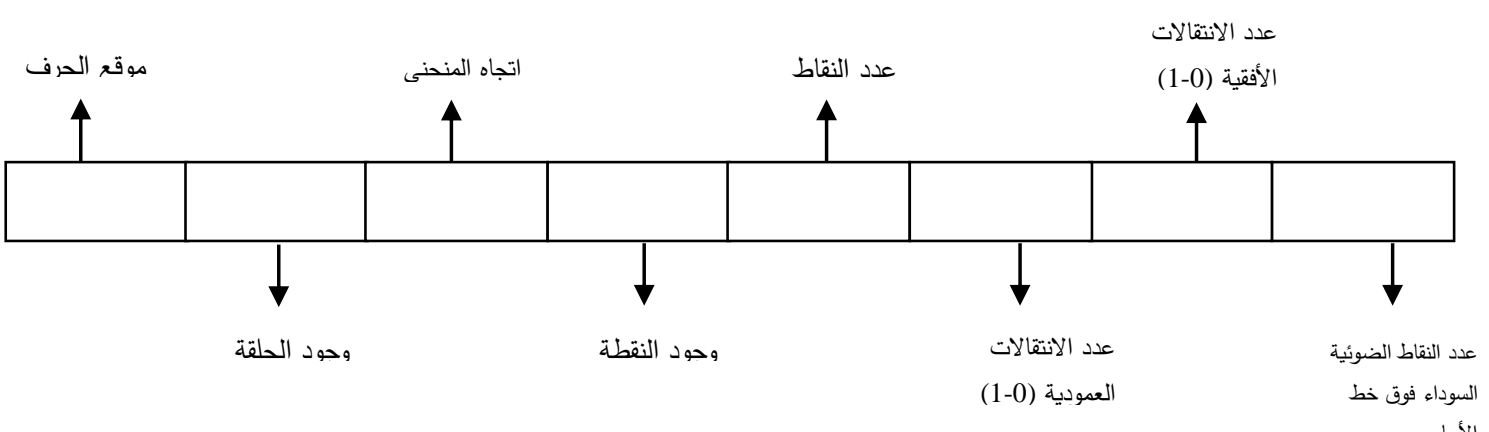

(الثكل-5) عناصر متجه السمات

وبعد إيجاد متجه السمات لكل حرف وبجميع أشكاله تتكون أربعة جداول للسمات حسب موقع الحرف في

تم تصميم أربعة نماذج لتمييز النص العربي المطبوع حسب موقع الحرف في الكلمة (ابتدائي، وسطي، نهائي، أو 
ونوع نموذج ماركوف المستخدم هو نموذج اليسار -إلى -اليمين المتوازي (parallel left-to-right) الذي ينسجم

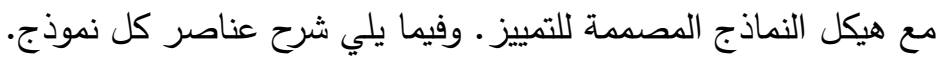

6-1-6 عناصر نموذج ماركوف الخفي المصم للحرف الابتدائي

لقد تم تصميم النموذج بحيث يضم تسع حالات يوضحها الثكل-6.

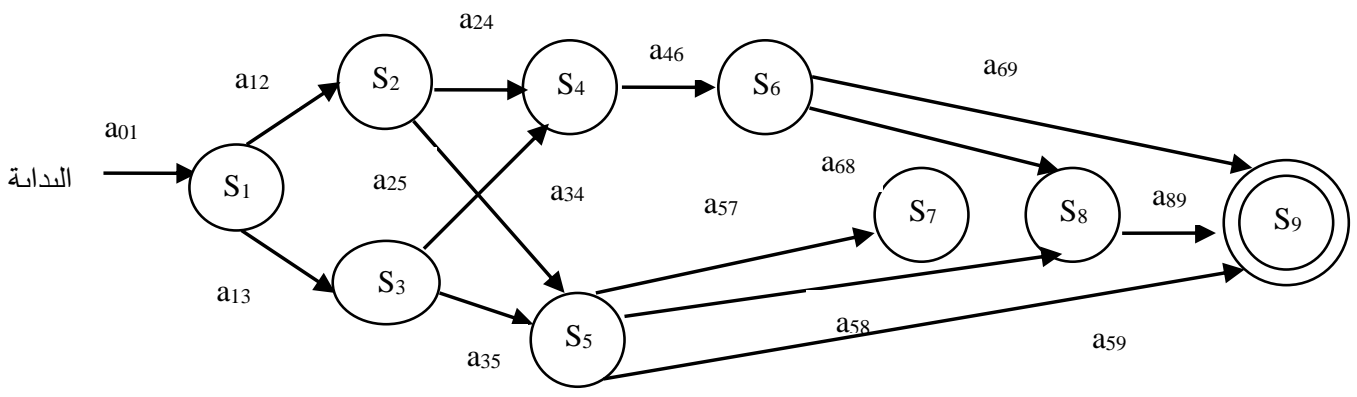

(الثكل-6) نموذج ماركوف الخفي للحرف الابتائي- تسع حالات

وكانت عناصر نموذج ماركوف الخفي للحرف الابتدائي كما يلي:

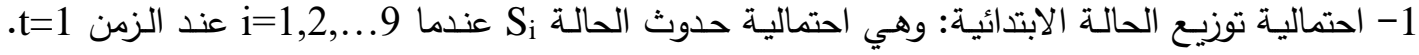

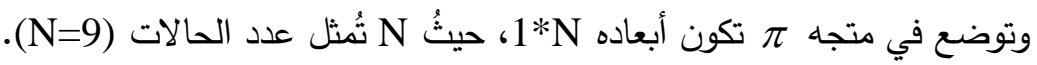

$\pi=\left[\begin{array}{lllllllll}1.0 & 0 & 0 & 0 & 0 & 0 & 0 & 0 & 0\end{array}\right]$

2- مصفوفة احتمالية الانتقال بين الحالات A حجمها N*N وحسب النموذج المصمم كان حجمها 9*9،

حيث ان N تمثل عدد حالات نموذج ماركوف المصدم. والجدول -1 يوضح قيم المصفوفة A A (الجدول -1) احتمالية الانتقال بين الحالات للحرف الابتدائي

\begin{tabular}{|c|c|c|c|c|c|c|c|c|c|}
\hline State & $\mathrm{S}_{1}$ & $\mathrm{~S}_{2}$ & $\mathrm{~S}_{3}$ & $\mathrm{~S}_{4}$ & $\mathrm{~S}_{5}$ & $\mathrm{~S}_{6}$ & $\mathrm{~S}_{7}$ & $\mathrm{~S}_{8}$ & $\mathrm{~S}_{9}$ \\
\hline $\mathrm{S}_{1}$ & $\mathbf{0}$ & $\mathbf{0 . 3 6 3 6}$ & $\mathbf{0 . 6 3 6 4}$ & $\mathbf{0}$ & $\mathbf{0}$ & $\mathbf{0}$ & $\mathbf{0}$ & $\mathbf{0}$ & $\mathbf{0}$ \\
\hline $\mathrm{S}_{2}$ & $\mathbf{0}$ & $\mathbf{0}$ & $\mathbf{0}$ & $\mathbf{0 . 5}$ & $\mathbf{0 . 5}$ & $\mathbf{0}$ & $\mathbf{0}$ & $\mathbf{0}$ & $\mathbf{0}$ \\
\hline $\mathrm{S}_{3}$ & $\mathbf{0}$ & $\mathbf{0}$ & $\mathbf{0}$ & $\mathbf{0 . 6 4 2 9}$ & $\mathbf{0 . 3 5 7 1}$ & $\mathbf{0}$ & $\mathbf{0}$ & $\mathbf{0}$ & $\mathbf{0}$ \\
\hline $\mathrm{S}_{4}$ & $\mathbf{0}$ & $\mathbf{0}$ & $\mathbf{0}$ & $\mathbf{0}$ & $\mathbf{0}$ & $\mathbf{1 . 0}$ & $\mathbf{0}$ & $\mathbf{0}$ & $\mathbf{0}$ \\
\hline $\mathrm{S}_{5}$ & $\mathbf{0}$ & $\mathbf{0}$ & $\mathbf{0}$ & $\mathbf{0}$ & $\mathbf{0}$ & $\mathbf{0}$ & $\mathbf{0 . 2 2 2 2}$ & $\mathbf{0 . 2 2 2 2}$ & $\mathbf{0 . 5 5 5 6}$ \\
\hline $\mathrm{S}_{6}$ & $\mathbf{0}$ & $\mathbf{0}$ & $\mathbf{0}$ & $\mathbf{0}$ & $\mathbf{0}$ & $\mathbf{0}$ & $\mathbf{0}$ & $\mathbf{0 . 3 0 7 7}$ & $\mathbf{0 . 6 9 2 3}$ \\
\hline $\mathrm{S}_{7}$ & $\mathbf{0}$ & $\mathbf{0}$ & $\mathbf{0}$ & $\mathbf{0}$ & $\mathbf{0}$ & $\mathbf{0}$ & $\mathbf{0}$ & $\mathbf{0}$ & $\mathbf{1 . 0}$ \\
\hline $\mathrm{S}_{8}$ & $\mathbf{0}$ & $\mathbf{0}$ & $\mathbf{0}$ & $\mathbf{0}$ & $\mathbf{0}$ & $\mathbf{0}$ & $\mathbf{0}$ & $\mathbf{0}$ & $\mathbf{1 . 0}$ \\
\hline $\mathrm{S}_{9}$ & $\mathbf{0}$ & $\mathbf{0}$ & $\mathbf{0}$ & $\mathbf{0}$ & $\mathbf{0}$ & $\mathbf{0}$ & $\mathbf{0}$ & $\mathbf{0}$ & $\mathbf{1 . 0}$ \\
\hline
\end{tabular}

يليها حساب التوزيع الاحتمالي لمسار رموز مشاهدات الحروف في مواقعها الأخرى. حيث كانت مصفوفة التوزيع الاحتمالي لرموز المشاهدات B (حجمها N*M) وحسب بيانات التدريب 17*9 حيث ان N تمثل عدد حالات نموذج ماركوف المصمم وان تمثل عدد رموز المشاهدات المتوقعة عند كل حالة، كما في الجدول-2 حيث

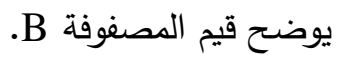


(الجدول -2) التوزيع الاحتمالي لرموز المشاهدات- الحرف الابتدائي

\begin{tabular}{|c|c|c|c|c|c|c|c|c|c|c|c|c|c|c|c|c|c|}
\hline$S^{0}$ & 1 & 2 & 3 & 4 & 5 & 6 & 7 & 8 & 9 & 10 & 11 & 12 & 13 & 14 & 15 & 16 & 17 \\
\hline$S_{1}$ & 1.0 & 0 & $\mathbf{0}$ & 0 & 0 & $\mathbf{0}$ & $\mathbf{0}$ & $\mathbf{0}$ & 0 & $\mathbf{0}$ & 0 & 0 & 0 & 0 & 0 & 0 & 0 \\
\hline $\mathbf{S}_{2}$ & $\mathbf{0}$ & 0.375 & \begin{tabular}{|l|l|} 
\\
\end{tabular} & 0.25 & 0 & 0 & $\mathbf{0}$ & 0 & 0 & $\mathbf{0}$ & 0 & 0 & $\mathbf{0}$ & $\mathbf{0}$ & 0 & 0 & 0 \\
\hline $\mathrm{S}_{3}$ & 0 & 0 & 0.6429 & 0.1429 & 0.1429 & \begin{tabular}{|l|}
0.0713 \\
\end{tabular} & 0 & 0 & 0 & 0 & 0 & 0 & 0 & 0 & 0 & 0 & 0 \\
\hline $\mathbf{S}_{4}$ & 0 & 0 & 0 & 0 & 0 & 0 & 0.7692 & 0.2308 & 0 & 0 & 0 & 0 & 0 & 0 & 0 & 0 & 0 \\
\hline $\mathbf{S}_{5}$ & 0 & 0 & 0 & 0 & O & 0 & 0 & $\mathbf{0}$ & 1.0 & 0 & 0 & 0 & 0 & 0 & 0 & 0 & 0 \\
\hline$S_{6}$ & 0 & 0 & 0 & $\mathbf{0}$ & 0 & 0 & $\mathbf{0}$ & 0 & $\mathbf{0}$ & 0.6154 & 0.2308 & \begin{tabular}{|l|}
0.1538 \\
\end{tabular} & 0 & 0 & 0 & $\mathbf{0}$ & $\mathbf{0}$ \\
\hline $\mathbf{S}_{7}$ & $\mathbf{0}$ & $\mathbf{0}$ & $\mathbf{0}$ & $\mathbf{0}$ & $\mathbf{0}$ & $\mathbf{0}$ & $\mathbf{0}$ & $\mathbf{0}$ & $\mathbf{0}$ & $\mathbf{0}$ & $\mathbf{0}$ & 0 & 0.5 & 0.5 & $\mathbf{0}$ & $\mathbf{0}$ & $\mathbf{0}$ \\
\hline $\mathbf{S}_{8}$ & 0 & 0 & 0 & 0 & 0 & 0 & 0 & 0 & 0 & 0 & 0 & 0 & 0 & 0 & 0.5 & 0.5 & 0 \\
\hline $\mathbf{S}_{9}$ & 0 & 0 & 0 & 0 & 0 & 0 & 0 & 0 & 0 & 0 & 0 & 0 & 0 & 0 & 0 & 0 & 1.0 \\
\hline
\end{tabular}

بالطريقة نفسها تم تصميم نموذج ماركوف الخفي للحرف الوسطي والنهائي والمنفصل وحساب عناصر النموذج وعناصر النموذج هي:

1- احتمالية توزيع الحالة الابتدائية

2- مصفوفة احتمالية الانتقال بين الحالات A

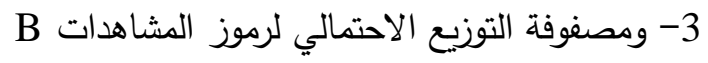

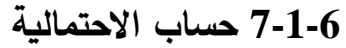

بعد استخلاص المشاهدات وتوليد متسلسلة المشاهدات لكل حرف حسب موقعه في الكلمة، تم تطبيق

الخوارزمية الأمامية (forward algorithm) لحساب احتمالية المشاهدات ، حيث تعمل الخوارزمية الأمامية على ولى حساب احتمالية المشاهدات وذلك بجمع احتمالات مسارات جميع الحالات الخفية التي بإمكانها أن تتتج متسلسلة المشاهدات، وطُبِت على بيانات التدريب لحساب احتمالية متسلسلة المشاهدات O لكل الحروف عند وجود النماذج

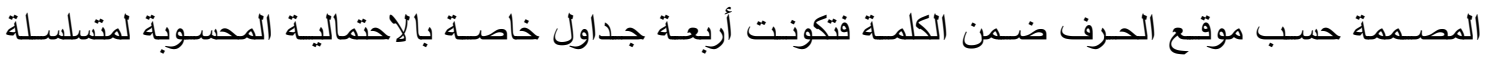
المشاهدات لكل حرف حسب موقعه في الكلمة، ندرج منها الجدول-3 الخاص بالحرف الابتدائي. (الجدول-3) نموذج من نتائج تطبيق الخوارزمية الأمامية للحرف الابتدائي

\begin{tabular}{|c|c|}
\hline الاحتمالية المحسوبة للحرف الابتدائي & 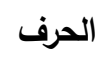 \\
\hline 0.007235288619995 & بـ \\
\hline 0.05878829956054 & ت \\
\hline 0.03919219970703 & $\dot{H}$ \\
\hline 0.007235288619995 & ج \\
\hline 0.0230809432983398 & $\boldsymbol{\sim}$ \\
\hline 0.024117469787598 & $\dot{~}$ \\
\hline 0.07792207792208 & 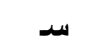 \\
\hline
\end{tabular}

كما تم حساب الجدول أعلاه للحرف بمواقعه الأخرى ( الوسطي، النهائي، والمنفصل) كمتطلب للخوارزمية الأمامية. 2-6 مرحلة الاختبار

لغرض اختبار كفاءة النموذج المصمم في التمييز تطبق الخطوات الآتية: 
تم اختيار صورة السطر النصي الموضحة في الثكل-7 كمثال لتطبيق نظام التمييز المُقترح عليها.

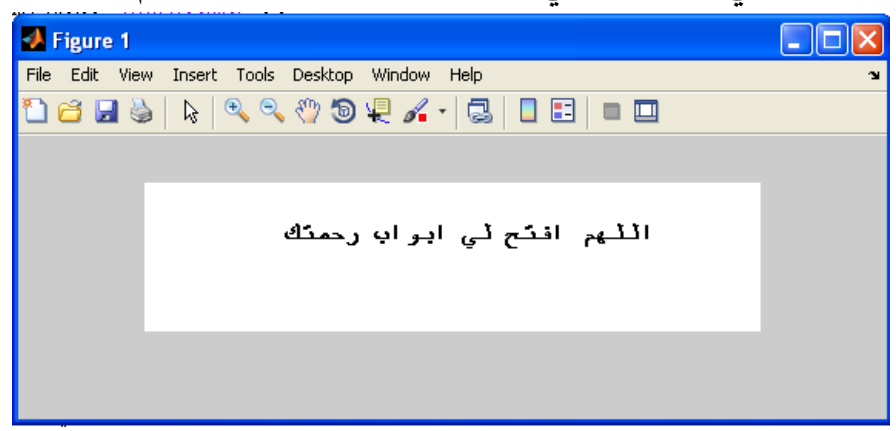

(الثكل-7) صورة السطر النصي المدخل

عملية الإدخال تبدأ بطباعة سطر نصي في برنامج معالج النصوص (Microsoft word 2003) استخدم خط من نوع Simplified Arabic fixed بحجم 18، ومن ثم خزنه على شكل صورة ثنائية في برنامج

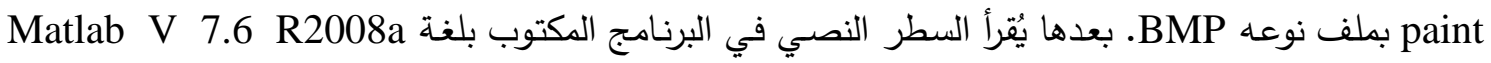
ويخزن في مصفوفة ثنائية. 2-2-6 تقطيع السطر النصي إلى مقاطع

يقطع السطر النصي في هذه الخطوة إلى المقاطع المكونة له، وبعد تحديد قيمة عمود البداية والنهاية لكل مفطع يتم خزنها للاستفادة منها في خطوة التقطيع إلى حروف. وناتج هذه الخطوة هو الحصول على الدقاطع

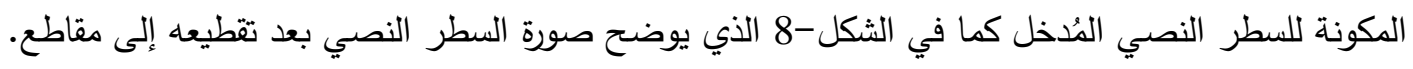

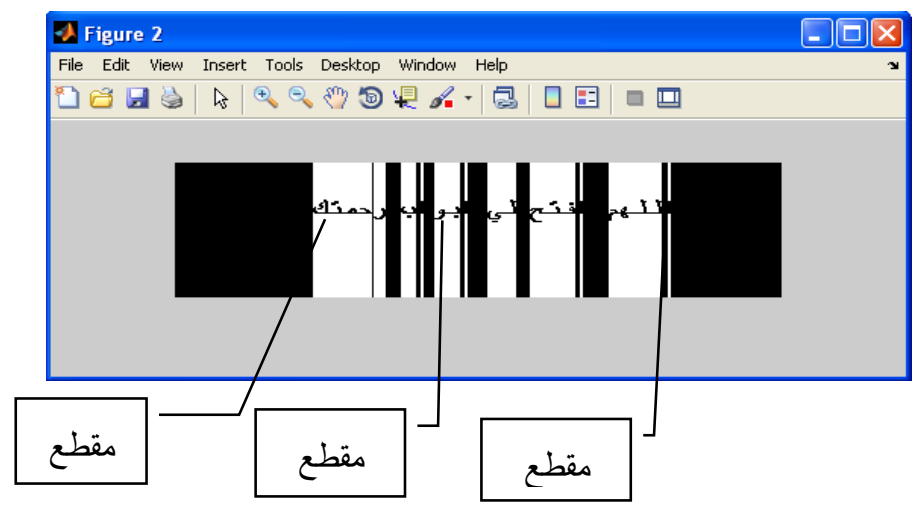

(الثكل-8) صورة السطر النصي بعد تقطيعه إلى مقاطع

3-2-6 تقطيع المقاطع إلى حروف

يتم تقطيع كل مقطع تم الحصول عليه من الخطوة السابقة إلى الحروف المكونة له بعد تحديد عمود بداية ونهاية كل حرف. وناتج هذه الخطوة يوضح في الثكل-9 حيثُ يتم تحويل نقطة سوداء واحدة إلى بيضاء لتكوين فراغات تفصل بين الحروف المقطعة. 


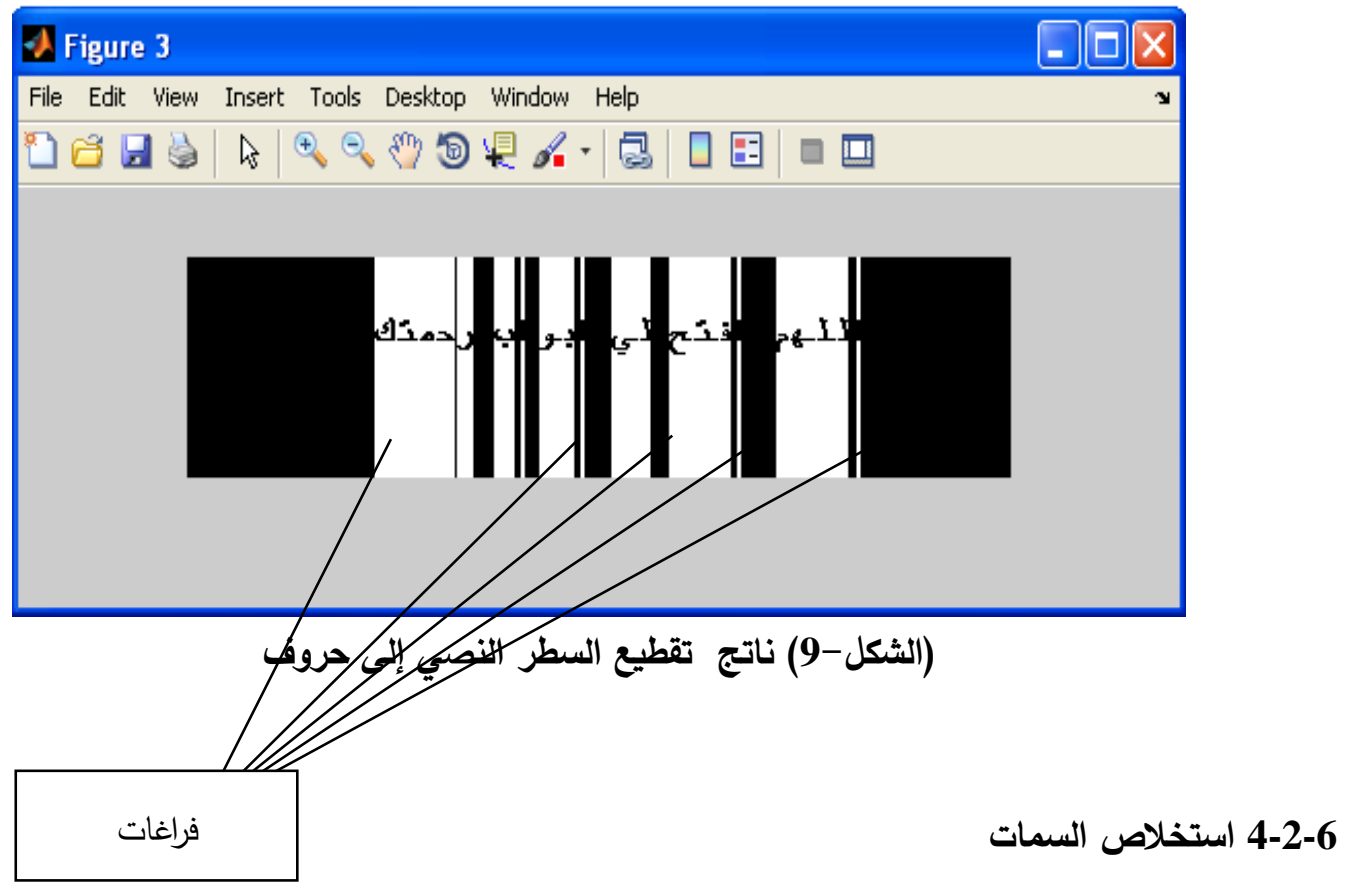

وبعد إكمال عملية التقطيع (segmentation) والوصول إلى الحروف المكونة للسطر النصي تم تحديد

عمود البداية والنهاية لكل حرف، وبذلك تحددت مساحة العمل على الحرف. يتم إجراء هذه الخطوة والخطوات اللاحقة على كل حرف في المقطع ومن اليمين إلى اليسار ولكل مقطع في السطر النصي، وهكذا يتم استخلاص سمات الحرف.

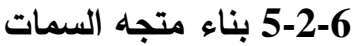

وعند تطبيق خوارزميات استخلاص السمات حصلنا على سمات كل حرف في السطر النصي المُدخل،

حيث يتم خزن هذه السمات في متجه السمات الخاص بكل حرف. وكانت قيم متجه السمات للسطر النصي المُدخل موضحة في الجدول-4.

(الجدول-4) متجه السمات لجزء من السطر النصي المُدخل

\begin{tabular}{|c|c|c|c|c|c|c|c|c|}
\hline \multirow[t]{2}{*}{ الحرف } & \multicolumn{8}{|c|}{ متــجه العــــات } \\
\hline & موقع الحرف | & وجود الحلقة & اتجاه المنحني & وجود النقطة & عدد النقاط & الالتقالات & الأنقالات & عدد النقاط الضوئية السوداء \\
\hline$\perp$ & 2 & $\mathbf{0}$ & 1 & $\mathbf{0}$ & $\mathbf{0}$ & 2 & 1 & 20 \\
\hline$t$ & 2 & 1 & $\mathbf{0}$ & $\mathbf{0}$ & $\mathbf{0}$ & 1 & 2 & 10 \\
\hline م & 3 & 0 & 1 & $\mathbf{0}$ & $\mathbf{0}$ & 1 & 1 & 6 \\
\hline 1 & 4 & 4 & $\mathbf{0}$ & $\mathbf{0}$ & $\mathbf{0}$ & 1 & 1 & 16 \\
\hline ف & 1 & 2 & $\mathbf{0}$ & 1 & 1 & 3 & 1 & 17 \\
\hline ت & 2 & 0 & 1 & 1 & 2 & 2 & 1 & 20 \\
\hline$\tau$ & 3 & $\mathbf{0}$ & 3 & $\mathbf{0}$ & $\mathbf{0}$ & 2 & 1 & 19 \\
\hline$\lrcorner$ & 1 & $\mathbf{0}$ & 1 & $\mathbf{0}$ & $\mathbf{0}$ & 2 & 1 & 20 \\
\hline ي & 3 & $\mathbf{0}$ & 2 & 2 & 2 & 2 & 2 & 2 \\
\hline
\end{tabular}


يتم في هذه الخطوة توليد متسلسلة المشـاهدات لكل حرف. وذلك بتحويل السمات المستخلصـة إلى الى متسلسلة من الرموز ، حيث يتم استدعاء الدالة الخاصـة بتوليد متسلسلة المشاهدات لكل حرف في السطر النصي وندي المُدخل. ويوضح الجدول-5 نموذج من متسلسلة المشاهدات لحروف السطر النصي المُدخل (الجدول-5) نموذج من متسلسلة المشاهدات لحروف السطر النصي المُّخل

\begin{tabular}{|c|c|c|c|c|c|}
\hline الحرف & \multicolumn{5}{|c|}{ متسلسلة المشاهدات } \\
\hline$\perp$ & 1 & 3 & 9 & 13 & - \\
\hline$t$ & 1 & 2 & 9 & 14 & 15 \\
\hline م & 1 & 3 & 9 & 14 & - \\
\hline 1 & 1 & 5 & 9 & - & - \\
\hline ف & 1 & 3 & 7 & 10 & - \\
\hline ت & 1 & 3 & 7 & 11 & - \\
\hline$\tau$ & 1 & 5 & 9 & 14 & - \\
\hline 」 & 1 & 3 & 9 & 15 & - \\
\hline
\end{tabular}

7-2-6 التمييز باستخدام نموذج ماركوف الخفي

بعد الحصول على متسلسلة المشاهدات للحرف يتم إدخالها إلى نموذج ماركوف الخفي المُقابل لموقع

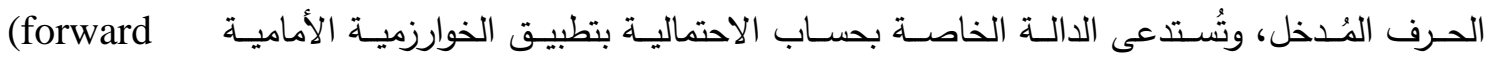
algorithm) التمييز باستخدام الخوارزمية الأمامية يتم استـعاء الدالة الخاصـة بالخوارزمية الأمامية لتحسب احتماليـة متسلسلة مشـاهدات الحرف المُدخل

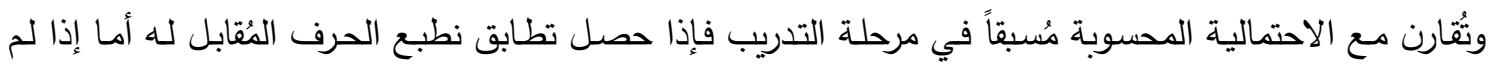
يحصل التطابق فيطبع عبارة حرف غير معروف. نُطبق هذه الخطوة على جميع الحروف المكونة للنص المُدخل.

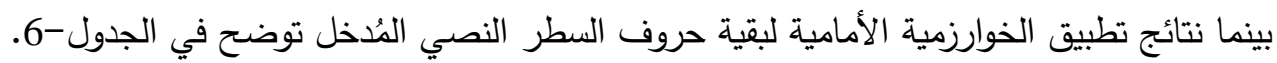
(الجدول-6) الاحتمالية عند تطبيق الخوارزمية الأمامية لحروف السطر المُدخل

\begin{tabular}{|c|c|}
\hline الاحتمالية المحسوبة & الحرف \\
\hline 0.0638542175229297 & $\perp$ \\
\hline 0.005050420761108 & 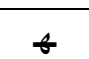 \\
\hline 0.037048339843750 & م \\
\hline 0.015872955322266 & 1 \\
\hline 0.156768798828125 & ف \\
\hline $\mathbf{0 . 0 5 4 7 5 2 3 4 9 8 5 3 5 1 6}$ & ت \\
\hline 0.031698226928711 & $\tau$ \\
\hline
\end{tabular}

وناتج تمييز صورة النص المُدخل بتطبيق الخوارزمية الأمامية موضحاً في الثكل-10. 


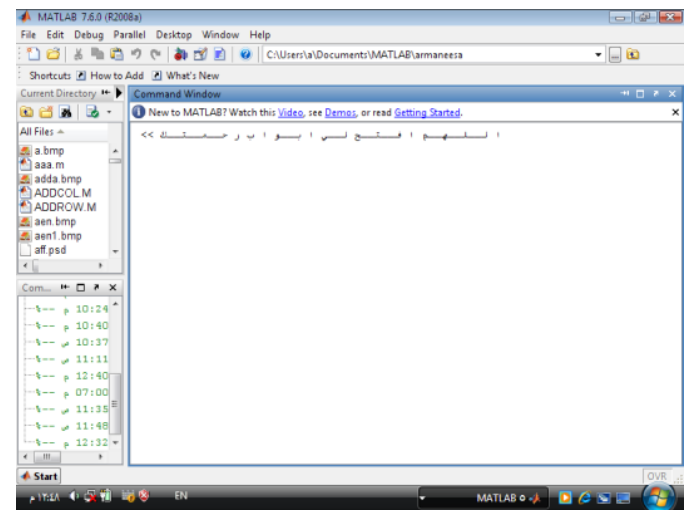

(الشكل-10) ناتج تمييز صورة النص المدخل بتطبيق الخوارزمية الأمامية

ومن خلال متابعة نتائج النظام في تمييز حروف 20 سطرا نصيا تضمنت اغلب حروف اللغة العربية وقد بلغت دقة التمييز (94.9\%) للحروف ذات الحجم والنمط الخطي الواحد، مما يجعل منـه أساس عمل أو لبنة أولى حروف لبناء نظام تمييز حروف مختلفة الأنماط والأحجام. 10- الاستنتاجات

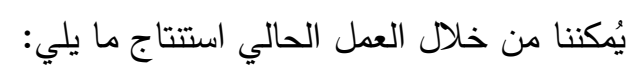

1- قدرة نموذج مـاركوف الخفي المصمم على التعرّف بسرعة محسوسـة وأداء عاليين كما مُبـين من خـلال متابعة أداء النظام في التعرّف على السطر النصي المُدخل الذي تم توضيحه في المقطع السابق.

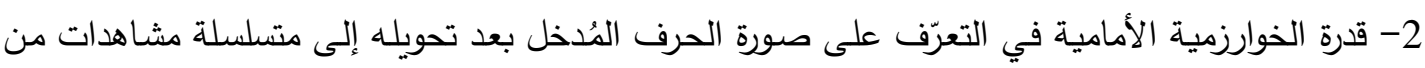

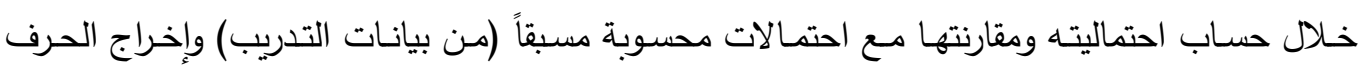
المقابلة احتماليته لاحتمالية الحرف المدخل.

هنالك عدة اقتراحات لتحسين أداء النظام وهي:

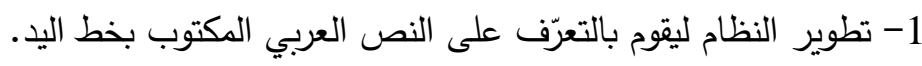

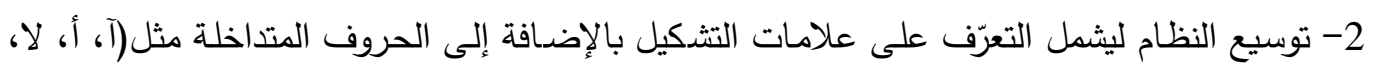
لآ، لاء، لاء). 3- تطوير النظام ليُطبق على أنماط مختلفة من الخطوط والأحجام. لأ.

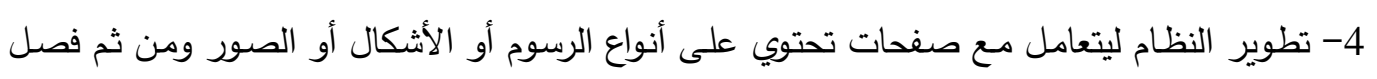
الصور والأشكال عن النص والتعامل معاه بصورة مستقلة. 5- التعرّف بدون التقطيع إلى حروف وذلك لتجاوز الأخطاء التي تُسبها مرحلة التقطيع. 


$$
\text { عجرش، آمال سفيح 2004، "استخدام المنظق المضبب آلية لتمييز الحروف العربية"، رسالة ماجستير }
$$

[2] Sharma Amit Kumar and kishor Mr.R Rama, 2007, "pattern recognition: Different available approaches", proceeding of National conference on challenges \& opportunities in information technology (COIT-2007) RIMT-IET, Mandi Gobindrh. www.rimtengg.com/coit 2007/.../coitindex.html

[3] Jain Anil K., Duin Robert P.W. and Mao Jain chang, 2000, "statistical pattern recognition: A review", IEEE Transaction pattern analysis and Machine intelligence, vol.22, No.1.

[4] Jannoud, Ismael Ahmed, 2007, "Automatic Arabic Handwritten Text Recognition System", American Journal of Applied sciences 4(11): 857-864, ISSN 1546-9239.

[5] Jurafsky Daniel and Martin James H., 2006, "speech and language processing: An introduction to natural language processing, computational linguistics and speech recognition", $2^{\text {nd }}$ Ed., prentice-Hall 2000, ISBN: 0-13-095069-6.

[6] Rabiner Lawrence R., 1989, "A Tutorial on Hidden Markov Models and selected Applications in speech recognition", proceedings of the IEEE, vol.77, NO.2.

$$
\begin{aligned}
& \text { الكيم، سلوان تحسين فالح 2005، "تصميم نظام لتمييز الحروف العربية باستخدام الخوارزميات الجينية"، } \\
& \text { رسالة ماجستير غير منشورة، قسم علوم الحاسبات، كلية العلوم، جامعة البصرة، العراق. } \\
& \text { الكسو، ابتهاج عبد الحميد محمد 2005، "استخدام الثبكات العصبية في تقدير رتب سلاسل مـاركوف }
\end{aligned}
$$

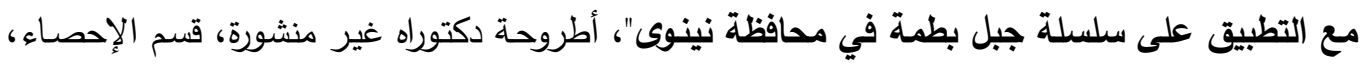

$$
\begin{aligned}
& \text { كلية علوم الحاسبات والرياضيات، جامعة الموصل، العراق. }
\end{aligned}
$$

[9] Sofia, Fatin Basher Abdul Ahad 2003, "An Implementation of Arabic speech recognition", Unpublished Ph.d. Thesis, Department of mathematical science, college of computer and mathematical science, university of Mosul, IRAQ.

[10] Aazami, Farshideh Einsele 2008, "Recognition of ultra low resolution, Antialiased text with small font sizes", Unpublished Ph.d. thesis, Scientarium informaticarum, Faculty of science, University of Fribourg, Switzerland.

[11] Dunham, Margaret H., 2002, "Data Mining introductory and advanced Topics", prentice Hall.

[12] Li xiaolin, Parizeau Marc and plamondon Rejean, 2000, "Training Hidden Markov Models with multiple observations-A combinational Method", IEEE Transactions on PAMI, vol.PAMI-22, NO.4, pp.371-377. 
[13] Attaluri, srilatha, 2007, "Detecting Meta Morphic Viruses using profile Hidden Markov Models", Unpublished M.Sc. thesis, computers science, the faculty of the department of computer science, university of San Jose State.

[14] Jecheva Vaselina, 2006, "A bout some Application of Hidden markov Model in intrusion detection system", International conference and computer systems and Technologies-compsys tech'06.

[15] Khorsheed M.S., 2003, "Recognizing handwritten Arabic Manuscripts using a single Hidden markov Model", Pattern Recognition letters 24. 